\title{
RELAÇÕES MATERNO-FILIAIS E SUA INFLUÊNCIA NO PESO PRÉ-DESMAMA DE ANIMAIS NELORE DA BAHIA*
}

\author{
MOTHER-OFFSPRING RELATIONS AND ITS INFLUENCE IN PREWEANING WEIGHT IN \\ NELLORE ANIMALS OF THE BAHIA
}

\author{
Souza, E.A. ${ }^{1}$, M.V. Andrea ${ }^{2}$, C.S. Santos ${ }^{2}$, M.J.R. Paranhos da Costa ${ }^{3,4}$, \\ T.C.B.S.C. Bittencourt ${ }^{5}$ e C.R. Marcondes ${ }^{6}$
}

\begin{abstract}
${ }^{1}$ Universidade Federal do Recôncavo da Bahia. UFRB. Cruz das Almas-BA. CEP 44380-000. Brasil. ema_aragao@yahoo.com.br

${ }^{2}$ Centro de Ciências Agrárias, Ambientais e Biológicas da Universidade Federal do Recôncavo da Bahia. UFRB. Cruz das Almas-BA. Brasil. mvander@ufba.br; claudiassufrb@hotmail.com

${ }^{3}$ Departamento de Zootecnia. Faculdade de Ciências Agrárias e Veterinárias. UNESP. Jaboticabal-SP. CEP 14884-900. Brasil.mpcosta@fcav.unesp.br

${ }^{4}$ ETCO. Grupo de Estudos e Pesquisas em Etologia e Ecologia Animal. Faculdade de Ciências Agrárias e Veterinárias. UNESP. Jaboticabal-SP. CEP 14884-900. Brasil.

${ }^{5}$ Escola de Medicina Veterinária de Universidade Federal da Bahia. UFBA. Salvador-BA. CEP 40170-110. Brasil. calmon@ufba.br

${ }^{6}$ EMBRAPA. Amazônia Oriental. C. Postal:48. CEP66095-100. Belém-PA. Brasil.cimarcon@cpatu.embrapa.br
\end{abstract}

\section{PaLAVRAS ChaVE ADICIONAIS \\ Comportamento animal. Desmama. Zebu.}

\section{RESUMO}

O presente trabalho objetivou avaliar as relações materno-filiais em animais Nelore $\mathrm{PO}$ (Puro de Origem) e Nelore CL (Cara Limpa), no Estado da Bahia. A composição genética não influenciou na LP (latência para ficar em pé) e apresentou valores médios de 71 e 52 minutos para o Nelore PO e Nelore CL, respectivamente. $\mathrm{Na}$ LPTM (latência para tentar mamar após fica em pé) foram observados valores médios de 39 minutos para o Nelore PO e 18 minutos para Nelore CL. Para variável TCC (tempo de contato com a cria), tanto as vacas $\mathrm{PO}$ quanto as $\mathrm{CL}$ obtiveram classe 4 (mantiveram em média de 60 a $80 \%$ de contato com a cria). O P120 (peso aos 120 dias), mostrou valores médios de $122,3 \mathrm{~kg}$ e 122,7 kg, respectivamente para PO e CL. Conclui-se que os comportamentos materno-filiais não sofreram diferenças em relação aos rebanhos para a maioria das variáveis estudadas. Os resultados deste

*Artigo parte da dissertação de Mestrado da $1^{\text {a }}$ autora.

Recibido: 7-2-07. Aceptado: 6-3-07.

\author{
AdDitionAl KEYWORDS \\ Animal Behavior. Weaning. Zebu.
}

estudo sugerem continuidade na pesquisa em busca de elementos que possam servir de parâmetro tecnológico a ser aplicado ao Estado da Bahia.

\section{SUMMARY}

The aim of this study was to investigate the maternal-offspring relationships in the bovine breeds Nellore PO (Pure Breed) and Nellore CL (Cara Limpa) in Bahia State. The genetic composition of breeds did not affect LP (latency time to stand); this variable showed mean values of $71 \mathrm{~min}$ and $52 \mathrm{~min}$, respectively, for Nellore PO and Nellore CL. The variable LPTM (latency time to begin suckling after standing) was significantly higher for Nellore PO (mean values of $39 \mathrm{~min}$ ) tham for Nellore CL (18 min). The variable TCC (time in contact with the calf) was classified as class 4 ( 60 to $80 \%$ of the time was spent with the calves). The P120 (weight at 120 days) had mean values of $122.3 \mathrm{~kg}$ and $122.7 \mathrm{~kg}$ respectively, for Nellore

Arch. Zootec. 58 (224): 729-732. 2009. 
PO and Nellore CL. In conclusion, the maternaloffspring behaviour had little influence of the genetic composition of the breeds. These results suggest that more studies are necessary to define technological parameters for cattle raising in Bahia State.

\section{INTRODUÇÃO}

O comportamento materno-filial em bovinos tem importância fundamental no melhoramento e preservação da espécie. Em uma abordagem psicoetológica sobre esse comportamento, Bussab (1998) relata sobre a necessidade da mãe permanecer junto às suas crias, a fim de garantir a tranqüilidade básica que irá favorecer o desenvolvimento e os estímulos diretos e indiretos influenciando nas habilidades e hábitos.

É claro que cada raça apresenta características intrínsecas ao seu comportamento, mas estas características sofrem alterações quando realizadas em diferentes ambientes. Assim, deve-se priorizar uma avaliação ambiental, levando-se em consideração o registro da temperatura e umidade do ar, além da ocorrência de chuvas (Paranhos da Costa e Cromberg, 1998).

A mãe tem papel decisivo na sobrevivência do filho, pois existe uma correlação positiva observada por Tokumaru (1998), entre os investimentos da mãe com o aumento na sobrevida e na taxa de reprodução destes, facilitando a adaptação desse ser às condições ambientes que lhes foram impostas.

O objetivo desse estudo foi investigar as relações materno-filiais em dois rebanhos bovinos do Estado da Bahia e sua influência no peso aos 120 dias de idade.

\section{MATERIAL E MÉTODOS}

O presente trabalho foi realizado em duas fazendas do Estado da Bahia, sendo a Fazenda Soraya (FS), no município de Serra Preta, com características climáticas de semiárido. A outra, a Fazenda de Pecuária da
Universidade Federal do Recôncavo da Bahia(UFRB), situada no município de Cruz das Almas, com características climáticas, tropical quente e úmida. Foram definidos dois rebanhos, o rebanho 1 composto por 9 (nove) vacas Nelore PO (puro de origem) da Fazenda Soraia (FS) e o rebanho 2 com 7 (sete) vacas Nelore CL (Cara Limpa) da Fazenda de Pecuária da Universidade Federal do Recôncavo da Bahia (UFRB). Em ambas, os rebanhos foram manejados em piquetes-maternidade (2 ha).

As medidas de comportameto foram realizadas por meio de observações diretas das atividades de vacas e bezerros nas primeiras horas após o parto. Utilizou-se amostragem focal e coleta contínua. Seguindo o modelo adaptado do etograma de trabalho (Paranhos da Costa et al., 1996).

Os registros terminaram logo após o bezerro conseguir realizar a primeira mamada ou, quando esta não ocorria, após seis horas de observação, sendo assim, atribuído o valor de 315 minutos para as latências (para ficar em pé e tentando mamar após ficar em pé).

\section{VARIÁVEIS ANALISADAS}

Para os bezerros foram consideradas quatro variáveis:

- Latência para ficar em pé (LP) - tempo que o bezerro levou desde o nascimento até permanecer apoiado nos quatro membros, estando ou não equilibrado;

- Latência para tentar mamar após ficar em pé (LPTM) - definido como o tempo que o bezerro levou após ficar em pé para iniciar a tentativa de apreensão dos tetos;

- Peso ao nascer (PN) - peso do bezerro no dia seguinte do nascimento;

- Peso aos 120 dias (P120) - peso do bezerro aos 120 dias de vida.

Para as vacas, a variável foi:

- Tempo em contato com a cria (TCC) porcentagem do tempo em que a vaca esteve em contato com a cria em relação ao tempo total de observação. 


\section{ANÁLISE ESTATÍSTICA}

Nas variáveis foi realizada análise de variância(ANOVA), utilizando o programa estatístico SAS versão 9.1 (SAS Institute Inc. 2002 - 2003), estimadas as médias, com seus respectivos desvios padrão e calculado o erro dessas variáveis. Após essa análise foi aplicado o teste de $\mathrm{F}$ ao nível de $5 \%$ de probabilidade para comparação de médias a fim de detectar as diferenças apresentadas no teste de F para os rebanhos 1 e 2, e para as variáveis LP, LPTM, P120.

A variável TCC foi classificada em classes: 1 - para mães que mantiveram contato de 0 a $20 \% ; 2$ - de 20 a 40\%; 3 - de 40 a $60 \%$; 4 - de 60 a $80 \%$ e 5 - acima de $80 \%$. Esses percentuais foram determinados a partir da relação do tempo total observado versus tempo em que a vaca esteve em contato com a cria.

\section{RESULTADOSEDISCUSSÃO}

No estudo, os dois grupos analisados apresentaram a maioria dos partos concentrados na classe 1 (de 0 a 10 m em relação ao rebanho), mas tal característica não influenciou no tempo em que as vacas dedicaram as crias, onde a variável TCC para ambos rebanhos obteve o valor 4 (de 60 a $80 \%$ de contato).

Em bovinos, o peso ao nascer tem influência decisiva na sobrevivência do bezerro. Conforme Schmidek(2003), bezerros muito leves tendem a apresentar menores índices de sobrevivência, sendo ideais pesos intermediários, ou seja, entre 30 e $35 \mathrm{~kg}$, como os obtidos neste trabalho e que podem ser observados na tabela I.

Agilidade do bezerro e cuidados que a mãe destina ao bezerro influenciarão na ingestão do colostro, que é de extrema importância para que o bezerro consiga consumir todos os nutrientes. Nutrientes estes que auxiliarão na sua qualidade de vida, além disso, também estará associado à manutenção de concentração sérica adequada das imunoglobulinas (Paranhos da Costa et al., 1996). Quanto mais esse neonato demora em levantar, mais tempo ele desprenderá para ingerir o colostro e possivelmente serão menos precoces (Kippert etal., 2006). Valores de LP variaram 28,0 a 119,0 minutos para as vacas do rebanho

Tabela I. Médias, desvios padrão (DP), mediana, valores máximos e mínimos para as características que apresentaram resultados significativos, com relação as raças analisadas. (Averages, standard deviations (DP), median, maximum and minimum values for the characteristics that showed significant results, regarding the analyzed breeds).

\begin{tabular}{cccccc}
\hline Característica & Média & DP & Mediana & Mínimo & Máximo \\
\hline PN (kg) & & & & & \\
Rebanho 1 & $33,00^{\mathrm{a}}$ & 3,51 & 33,20 & 26,00 & 38,00 \\
$\quad$ Rebanho 2 & $35,00^{\mathrm{a}}$ & 4,89 & 33,75 & 30,00 & 40,00 \\
LP (min.) & & & & & \\
$\quad$ Rebanho 1 & $71,00^{\mathrm{a}}$ & 33,38 & 73,50 & 28,00 & 119,00 \\
$\quad$ Rebanho 2 & $52,00^{\mathrm{a}}$ & 18,32 & 54,00 & 28,00 & 80,00 \\
LPTM (min.) & & & & & \\
$\quad$ Rebanho 1 & $39,00^{\mathrm{b}}$ & 11,39 & 39,00 & 21,00 & 53,00 \\
$\quad$ Rebanho 2 & $18,00^{\mathrm{a}}$ & 11,87 & 14,37 & 8,00 & 42,00 \\
P120 (kg) & & & & & \\
Rebanho 1 & $122,00^{\mathrm{a}}$ & 7,35 & 124,12 & 109,00 & 131,00 \\
Rebanho 2 & $123,00^{\mathrm{a}}$ & 7,89 & 126,50 & 107,00 & 131,00 \\
\hline
\end{tabular}

Médias seguidas pela mesma letra não diferem estatisticamente entre si pelo teste de $F, p<0,05$. 
1 , enquanto que as vacas rebanho 2 apresentaram valores de 28,0 a 80,0 minutos, como podem ser ressaltados na tabela I.

Dados obtidos por Toledo (2005), quando avaliou o comportamento nas raças Nelore e Guzerá, possibilitaram ver que não ocorreu um efeito significativo, fato que, possivelmente, esta relacionado a fatores inerentes ao bezerro, como tempo procurando tetos em outras partes do corpo da mãe ou mesmo quando permaneciam em pé com qualquer outra atividade antes de encontrar o úbere. Tal comportamento apresentou-se de maneira contrária em nossos estudos, onde o rebanho 2 demonstrou valores significativos quando relacionado ao rebanho 1, para a variável LPTM (tabela I).

Segundo Cubas et al. (2001), quando se pensa em seleção de animais, logo vem à mente a fase de cria, pois é uma das etapas

\section{BIBLIOGRAFIA}

Bussab, V.S.R. 1998. Uma abordagem psicoetológica do comportamento materno. In: Paranhos da Costa, M.J.R.; Cromberg, V.U. (Eds.). Comportamento materno em mamíferos: bases teóricas e aplicações aos ruminantes domésticos. Sociedade Brasileira de Etologia, São Paulo. pp. 17-30.

Cubas, A.C., D. Perotto, J.J.S. Abrahão e S.C. Mella. 2001. Desempenho até a desmama de bezerros Nelore e cruzas com Nelore. Rev. Bras. Zootecn., 30: 694-701.

Kippert, C.J., P.R.N. Rorato, L.T. Campos, A.A. Boligon, T. Weber, D.G. Gheller e J.S. Lopes. 2006. Efeito de fatores ambientais sobre escores de avaliação visual à desmama e estimativa do parâmetro genéticos, para bezerros da raça charolês. Ciência Rural, 36: 579-585.

Paranhos da Costa, M.J.R., V.U. Cromberg e J.H. Ardesh. 1996. Diferenças na latência da primeira mamada em quatro raças de bovinos de corte. In: Congresso de Zootecnia, 6, 1996. Actas do congresso...Associação Portuguesa dos Engenheiros Zootécnicos, 2: 343-348. Évora.

Paranhos da Costa, M.J.R. e V.U. Cromberg. 1998. Relações materno-filiais em bovinos de corte nas primeiras horas após o parto. In: Paranhos que mais influencia nos custos de produção e ganho de peso, como também está correlacionada a fatores de precocidade. Podemos ver que nos rebanhos analisados ocorreu uma homogeneidade nos resultados, onde os valores médios foram de 122,00 e $123,00 \mathrm{~kg}$, para os rebanho 1 e rebanho 2 , respectivamente (tabela I).

\section{CONCLUSÕES}

As diferenças nos rebanhos não influenciaram no comportamento maternofilial;

O rebanho 1 foi significativo para a maioria das variáveis analisadas, exceto para LPTM;

A partir dessa avaliação espera - se utilizar, de maneira mais precisa, técnicas de manejo que possam melhorar os índices de ganho de peso.

da Costa, M.J.R.; Cromberg V.U. (Eds.) Comportamento materno em mamíferos: bases teóricas e aplicações aos ruminantes domésticos. Sociedade Brasileira de Etologia. São Paulo. pp. 215-235.

SAS Institute INC. 2002 - 2003. Statistical analysis system. Release 9.1. (Software). Cary. USA.

Schmidek, A. 2003. Análises dos fatores genéticos e ambientais relacionados a características de vigor e qualidade materna para as raças Nelore e Guzerá. Dissertação (Mestrado em Genética e Melhoramento Animal). Faculdade de Ciências Agrárias e Veterinárias. UNESP. Jaboticabal.

Tokumaru, R.S. 1998. Bases evolutivas do comportamento materno. In: Paranhos da Costa, M.J.R.; Cromberg V.U. (Eds.) Comportamento materno em mamíferos: bases teóricas e aplicações aos ruminantes domésticos. Sociedade Brasileira de Etologia. São Paulo. pp. 9-16.

Toledo, L.M. 2005. Fatores intervenientes no comportamento de vacas e bezerros do parto até a primeira mamada. Tese (Doutorado em Genética e Melhoramento Animal). Faculdade de Ciências Agrárias e Veterinárias. UNESP. Jaboticabal. 